\title{
Bragg- and Moving-glasses: a theory of disordered vortex lattices
}

\author{
T. Giamarchi ${ }^{\mathrm{a} *}$ and P. Le Doussal ${ }^{\mathrm{b}} \dagger$ \\ ${ }^{\text {a} L a b o r a t o i r e ~ d e ~ P h y s i q u e ~ d e s ~ S o l i d e s, ~ U n i v e r s i t e ́ ~ P a r i s-S u d, ~ B a ̂ t ~ 510, ~} 91405$ Orsay, France \\ ${ }^{\mathrm{b}}$ CNRS-Laboratoire de Physique Théorique de l'Ecole Normale Supérieure, 24 rue Lhomond, F-75231 \\ Paris, France
}

\begin{abstract}
We study periodic lattices, such as vortex lattices in type II superconductors in a random pinning potential.
For the static case we review the prediction [1, 2] that the phase diagram of such systems consists of a topologically ordered Bragg glass phase, with quasi long range translational order, at low fields. This Bragg glass phase undergoes a transition at higher fields into another glassy phase, with dislocations, or a liquid. This proposition is compatible with a large number of experimental results on BSCCO or Thalium compounds. Further experimental consequences of our results and relevance to other systems will be discussed.

When such vortex systems are driven by an external force, we show that, due to periodicity in the direction transverse to motion, the effects of static disorder persist even at large velocity[3]. In $d=3$, at weak disorder, or large velocity the lattice forms a topologically ordered glass state, the "moving Bragg glass", an anisotropic version of the static Bragg glass. The lattice flows through well-defined, elastically coupled, static channels. We determine the roughness of the manifold of channels and the positional correlation functions. The channel structure also provides a natural starting point to study the influence of topological defects such as dislocations. In $d=2$ or at strong disorder the channels can decouple along the direction of motion leading to a "smectic" like flow. We also show that such a structure exhibits an effective transverse critical pinning force due to barriers to transverse motion, and discuss the experimental consequences of this effect.
\end{abstract}

\section{Introduction}

To understand the effects of static substrate disorder on periodic media such as vortex lattices [4] is of paramount importance both for the technological applications of high- $T_{c}$ materials and from a purely theoretical point of vue. Such a study also applies to many other physical problems such as charge density waves (CDW), Wigner crystals, colloids, magnetic bubbles.

For the vortex lattices it is generally agreed that disorder leads to a glass state with diverging barriers and pinning [5.6.6. Although the precise nature of the glass state has been the subject of much debate, most theories agreed on the absence of translational order. General arguments also tended to prove that disorder would always favor the presence of dislocations [5]. Many points were not naturally fitting in the framework of

\footnotetext{
*Laboratoire associated to CNRS; email: giam@lps.upsud.fr

†email: ledou@physique.ens.fr
}

these theories such as the existence of a first order transition [7,8], decoration experiments of showing remarkably large regions free of dislocations [9]. On the side of theory, scaling arguments 10 suggested, within a purely elastic decription, a slower, logarithmic, growth of deformations.

In a recent work we have obtained the first quantitative theory of the elastic vortex lattice [1] in presence of point disorder [11. Contrarily to previous approaches, it provides a description valid at all scales and demonstrates that while disorder produces algebraic growth of displacements at short length scales, periodicity takes over at large scales and results in a decay of translational order at most algebraic. Moreover we showed [1] using energy arguments that dislocations are not favorable for weak disorder in $d=3$. This implies the existence of a thermodynamic glass phase, as far as energy and very low current transport properties are concerned, retaining a nearly perfect (i.e. algebraic) translational order and thus Bragg peaks: the "Bragg glass". 


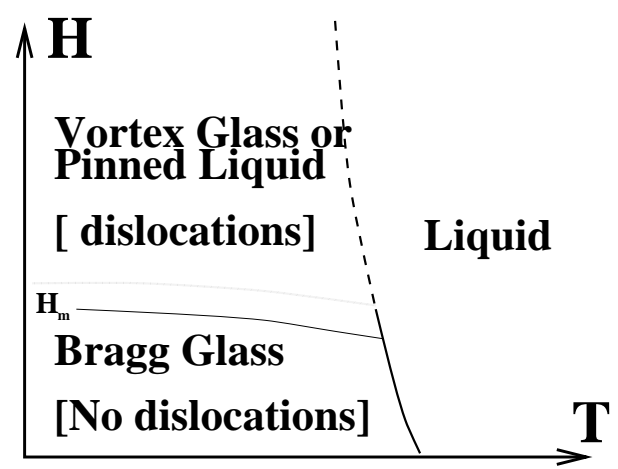

Figure 1. The stability region of the Bragg glass phase in the magnetic field $H$, temperature $T$ plane is shown schematically. The thick line is expected to be first order, whereas the dotted line should be either second order or a crossover. Upon increasing disorder the field induced melting occurs for lower fields as indicated by the thin solid line.

Because it retains a "lattice" structure and Bragg peaks, this glass phase is radically different from the vortex glass picture. We proposed [1] that the phase seen experimentally at low fields was the Bragg glass, solving the apparent impossibility of a pinned solid. This accounted naturally for the first-order transition and the decoration experiments. Our prediction [1] received subsequent further support both from numerical [12] or analytical [13,14] calculations.

Upon raising the field, or equivalently increasing the disorder, the Bragg glass should disappear by spontaneous generation of dislocations, when the translational length $R_{a}$ (the length for which relative displacements become of order of the lattice spacing $a$ ) is of the order of $a$. The critical point occuring on the melting line 15,8 was the end point of the transition line between the Bragg glass at low fields and a topologically disordered glassy phase (or a strongly pinned liquid) at higher field. The topology of the phase diagram proposed in [1] is as depicted in Fig. 1.

Several recent experiments can be interpreted to confirm the picture proposed in [1]. Neutron experiments can be naturally interpreted in term of the Bragg glass [16]. In BSCCO neutron peaks are observed at low fields and disappear upon raising the field [17]. The phase diagram of BSCCO [15] is also compatible with of our theory, the second magnetization peak line corresponding to the predicted field driven transition. A numerical estimate [2] of the melting field $H_{M}$ for BiSCCO gives $H_{M} \sim 400 G$ in good agreement with the observed experimental values [18]. This line is found to be relatively temperature independent at lower temperatures and to be shifted downwards upon increase of point disorder 18, 19. Similar types of phase diagrams are also observed in a variety of materials, including YBCO, organic superconductors and heavy fermion compounds. More experimental consequences and references can be found in [2].

Let us now turn to the dynamics of the driven system. It is crucial to determine how much of the glassy properties remain, and how translational and topological order behave [20]. Indeed several experiments suggest that a fast moving lattice is more ordered than a static one [21, 16]. Arguments [22 based on large velocity expansion 23] concluded that at low $T$ and above a certain velocity the moving lattice is a crystal at an effective temperature $T^{\prime}=T+T_{s h}$, with bounded displacements and no glassy properties.

We showed [3] that in fact the perturbation theory [22] breaks down, even at large $v$. Some modes of the disorder are not affected by the motion and the periodic structure driven along $x$ experiences a transverse static pinning force $F_{\text {stat }}$, perpendicular to the direction of motion.

$F_{\alpha}^{\text {stat }}(r, u)=V(r) \rho_{0} \sum_{K \cdot v=0} i K_{\alpha} \exp (i K \cdot(r-u))(1)$

where the random potential has correlations $\left\langle V(r) V\left(r^{\prime}\right)\right\rangle=\Delta\left(r-r^{\prime}\right)$ of range $r_{f}$. This force originates only from the periodicity along $y$ and the uniform density modes along $x$ [3,24]. It leads to a moving glass (MG).

We analyse here this result more quantitatively, using a functional RG to study the equation of motion. We only quote here the RG equation for the disorder term, for the periodic structure at 


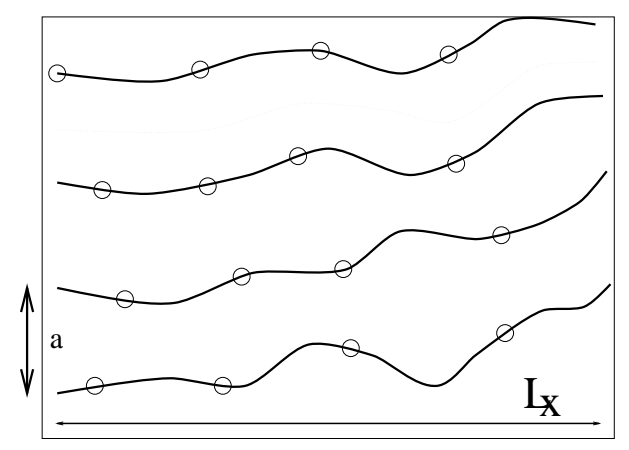

Figure 2. Motion in the moving glass occurs through static channels wandering at distance $a$ over lengths $L_{x} \sim L_{y}^{2}$. If dislocations are present $(d=2$ or strong disorder in $d=3)$ they should lead to a decoupling of channels, as indicated by the dotted line.

$T=0$ (for the complete calculation see [25])

$\frac{d \Delta(u)}{d l}=\Delta(u)+\Delta^{\prime \prime}(u)(\Delta(0)-\Delta(u))$

where a factor $\frac{1}{4 \pi v c} \epsilon$, with $\epsilon=3-d$ has been absorbed in $\Delta(u)$ (chosen to be of period 1). For $d>3$ disorder renormalizes to zero and the moving system is a crystal. For $d<3 \Delta$ flows to a new fixed point $\Delta^{*}(u)=\Delta(0)(l)+u^{2} / 2-u / 2$, showing that the static disorder is still relevant in the moving structure (with the same conclusion in $d=3$, 25]). The value of $\Delta(0)(l)$ grows unboundedly as $\Delta(0)(l)=\Delta(0) e^{\epsilon l}$ which indicates the existence of a random force along the $y$ direction, generated under renormalization. A similar force is generated along $x$ [25]. This does not spoil the above fixed point, since one can always separates $\Delta(0)$ and $\Delta(u)-\Delta(0)$. This has several consequences. In particular, for $d \leq 3$ the system remains a glass and motion occurs through elastic channels, as shown on figure 2. They are the easiest paths where particle follow each other in their motion. They form a manifold of elastically coupled, almost parallel lines or sheets (for vortex lines in $d=3$ ) directed along $x$ and characterized by some transverse wandering $u_{y}$. In the laboratory frame they are deter-

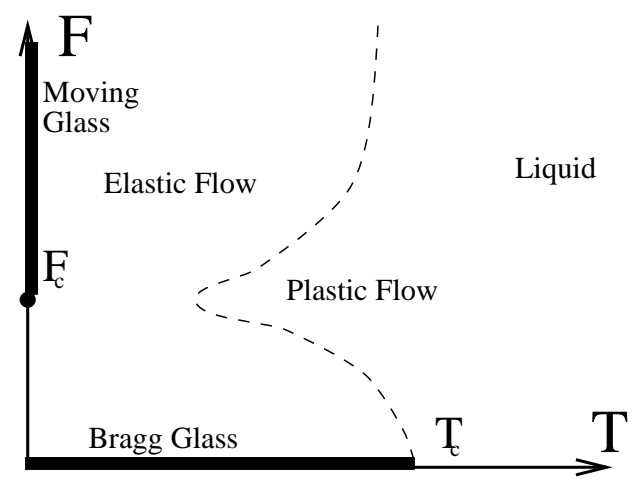

Figure 3. Phase diagram in force $F$, temperature $T$, for weak disorder and in $d=3$. At zero external force the system is in the free Bragg glass state. At large velocities, in the moving Bragg glass one. This suggests that in this case the depinning transition could be purely elastic.

mined by the static disorder and do not fluctuate with time. Such channels were subsequently observed in numerical simulations [26] and in recent decoration in motion experiments [27]. In $d=3$ diplacements only grow logarithmicaly, so the MG conserves quasi-long range translational order. Thus similarly to the statics, the MG in $d=3$ at weak disorder or large velocity is expected to retain perfect topological order. This leads to the $F-T$ phase diagram of figure 3 . In $d=2$ however displacements grow algebraically and dislocations are likely to appear. The existence of channels [3] then naturally suggests a scenario by which dislocations affect the MG: when the periodicity along $x$ is retained, e.g., presumably in $d=3$ at weak disorder, the channels are coupled along $x$. Upon increasing disorder or decreasing velocity in $d=3$, or in $d=2$, decoupling between channels can occur, reminiscent of static decoupling in a layered geometry 14]. Dislocations are then inserted between the layers, naturally leading to a flowing smectic glassy state, recently observed in $d=2$ numerical simulations 26]. Indeed, the transverse smectic order is likely to be more stable than topological order along $\mathrm{x}$, because of particle conservation [25]. 
As an important consequence of the existence of the MG, barriers for transverse motion exist once the pattern of channels is established. Thus the response to an additional small transverse force $F_{y}$ is very non linear with activated behaviour. At $T=0$ and neglecting the dynamic part of the disorder a true transverse critical current $J_{y}^{c}$ exists. This pinning force can directly be obtained from the RG equation (2). Since its fixed point has a non analycity at $u=0$, (leading to $\left.\Delta^{\prime}\left(0^{+}\right)=1 / 2\right)$ there is a critical force, determined at the Larkin length $L_{y}$ :

$F_{c}=\int d q G(q) \Delta^{\prime}\left(0^{+}\right)=\frac{\epsilon}{4 \pi v c_{y}} \sim L_{y}^{-2}$

(3) coincides with the more qualitative derivation consisting in balancing the pinning energy with the transverse Lorentz force acting on a Larkin domain [3]. Unlike in the $v=0$ case, the critical force does not kill the random force in the FRG equation. The MG is dominated by the competition between the random force and the critical force.

These predictions can be tested in experiments on the vortex lattice, or other systems such as colloids, magnetic bubbles or CDW, or or numerical simulations. Additional physical consequences and references can be found in [3.25].

\section{REFERENCES}

1. T. Giamarchi and P. Le Doussal, Phys. Rev. Lett. 72, 1530 (1994); Phys. Rev. B 52, 1242 (1995).

2. T. Giamarchi and P. Le Doussal, Phys. Rev. B 55, 6577 (1997).

3. T. Giamarchi and P. Le Doussal, Phys. Rev. Lett. 76, 3408 (1996).

4. G. Blatter et al., Rev. Mod. Phys. 66, 1125 (1994).

5. M. P. A. Fisher, Phys. Rev. Lett. 62, 1415 (1989); D. S. Fisher, M. P. A. Fisher, and D. A. Huse, Phys. Rev. B 43, 130 (1990).

6. M. Feigelman, V. B. Geshkenbein, A. I. Larkin, and V. Vinokur, Phys. Rev. Lett. 63, 2303 (1989).

7. M. Charalambous, J. Chaussy, and P. Lejay, Phys. Rev. B 45, 5091 (1992).
8. H. Safar et al., Phys. Rev. Lett. 70, 3800 (1993).

9. D. G. Grier et al., Phys. Rev. Lett. 66, 2270 (1991).

10. T. Nattermann, Phys. Rev. Lett. 64, 2454 (1990); J. Villain and J. F. Fernandez, Z Phys. B 54, 139 (1984).

11. S. E. Korshunov, Phys. Rev. B 48, 3969 (1993).

12. M. J. P. Gingras and D. A. Huse, Phys. Rev. B 53, 15193 (1996); S. Ryu, A. Kapitulnik, and S. Doniach, Phys. Rev. Lett. 77, 2300 (1996) and references therein.

13. J. Kierfeld, T. Nattermann, and T. Hwa, Phys. Rev. B 55, 626 (1997).

14. D. Carpentier, P. Le Doussal, and T. Giamarchi, Europhys. Lett. 35, 379 (1996).

15. E. Zeldov and al., Nature 375, 373 (1995).

16. U. Yaron and al., Phys. Rev. Lett. 73, 2748 (1994); T. Giamarchi and P. Le Doussal, Phys. Rev. Lett. 75, 3372 (1995).

17. R. Cubbit and al., Nature 365, 407 (1993).

18. B. Khaykovich and et al., Phys. Rev. Lett. 76, 2555 (1996).

19. N. Chikumoto and al., Physica C 185-189 2201 (1991); Phys. Rev. Lett. 69, 1260 (1992).

20. M. J. Higgins and S. Bhattacharya, Physica C 257, 232 (1996).

21. R. Thorel and al., J. Phys. (Paris) 34, 447 (1973).

22. A. E. Koshelev and V. M. Vinokur, Phys. Rev. Lett. 73, 3580 (1994).

23. A. Schmidt and W. Hauger, J. Low Temp. Phys 11, 667 (1973); A. I. Larkin and Y. N. Ovchinnikov, Sov. Phys. JETP 38, 854 (1974).

24. T. Giamarchi and P. Le Doussal, Phys. Rev. Lett. 78, 752 (1997).

25. T. Giamarchi and P. Le Doussal, 1997, to be published.

26. K. Moon and al., Phys. Rev. Lett. 77, 2378 (1997), S. Ryu et al. to be published.

27. M. Marchevsky and al., Phys. Rev. Lett. 78, 531 (1997). 